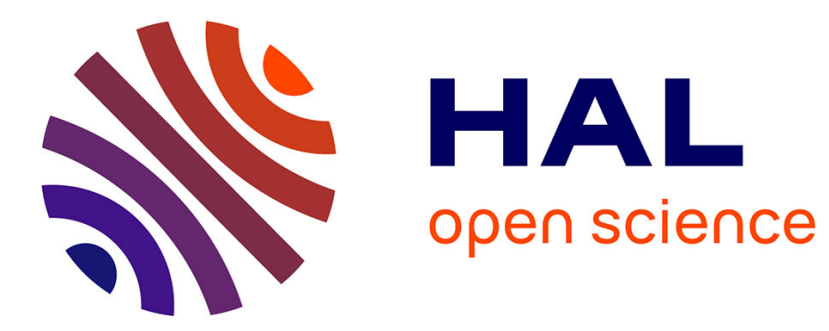

\title{
Opportunistic Random Access Scheme Design for OFDMA-based Indoor PLC Networks
}

\author{
Rongping Dong, Meryem Ouzzif, Samir Saoudi
}

\section{To cite this version:}

Rongping Dong, Meryem Ouzzif, Samir Saoudi. Opportunistic Random Access Scheme Design for OFDMA-based Indoor PLC Networks. IEEE Transactions on Power Delivery, 2012, 27 (4), pp. 2073 2081. 10.1109/TPWRD.2012.2203616 . hal-00739562

\section{HAL Id: hal-00739562 https://hal.science/hal-00739562}

Submitted on 8 Oct 2012

HAL is a multi-disciplinary open access archive for the deposit and dissemination of scientific research documents, whether they are published or not. The documents may come from teaching and research institutions in France or abroad, or from public or private research centers.
L'archive ouverte pluridisciplinaire HAL, est destinée au dépôt et à la diffusion de documents scientifiques de niveau recherche, publiés ou non, émanant des établissements d'enseignement et de recherche français ou étrangers, des laboratoires publics ou privés. 


\title{
Opportunistic Random-Access Scheme Design for OFDMA-Based Indoor PLC Networks
}

\author{
Rongping Dong, Student Member, IEEE, Meryem Ouzzif, Member, IEEE, and Samir Saoudi, Senior Member, IEEE
}

\begin{abstract}
Multiuser systems can benefit from multiuser diversity by assigning channels to users with better channel conditions at different time instants. In this paper, we present an opportunistic random-access scheme for orthogonal frequency-division multiple-access-based indoor power-line communication systems, based on time- and frequency-varying channel conditions, to exploit multiuser diversity. The proposed scheme dynamically adjusts the backoff time of each user according to its own channel-state variations in time and frequency domains during the contention procedure and, thus, "better" users have a higher priority to contend over their favorable subchannels. Moreover, subchannels are assigned to users with better channel conditions in order to further enhance the system throughput. In addition, an analytical throughput model for such a multiuser and multichannel system is derived to obtain the optimal parameter settings of the proposed access scheme. Simulation results show that the proposed scheme provides significant improvement in system throughput even in the case where the number of users highly exceeds that of subchannels.
\end{abstract}

Index Terms-Crosslayer optimization, multiuser diversity, opportunistic communications, orthogonal frequency-division multiple access (OFDMA), power-line communication (PLC).

\section{INTRODUCTION}

$\mathbf{P}$ OWER-LINE communication (PLC) systems become increasingly viewed as an attractive two-fold solution to the "last mile" and "last inch" access [1]. By employing the orthogonal frequency-division multiplexing (OFDM) and other techniques, PLC systems can attain up to a $150-\mathrm{Mb} / \mathrm{s}$ information rate [2] at the physical (PHY) layer, which makes PLC networks more appropriate to convey home multimedia applications and data services. As an extension of OFDM, orthogonal frequency-division multiple access (OFDMA) attracts great interest for multiple access since it inherits the main advantages of OFDM [i.e., high spectral efficiency and superior performance in the presence of intersymbol interference (ISI)]. In OFDMA systems, the whole frequency spectrum is divided into multiple narrowband subchannels, and multiple access is achieved by assigning subsets of subcarriers to individual users.

Manuscript received November 15, 2011; accepted May 22, 2012. Date of publication September 04, 2012; date of current version September 19, 2012. Paper no. TPWRD-00977-2011.

R. Dong was with the Department of Signal and Communications, Telecom Bretagne, Brest, France, and Orange Labs, Lannion, France. She is now with Lab-STICC, Université de Bretagne-Sud, Centre de recherche, Lorient 56321, France (e-mail: rongping.dong@univ-ubs.fr).

M. Ouzzif is with Orange Labs, Cesson-Sevigne 35512, France (e-mail: meryem.ouzzif@orange.com).

S. Saoudi is with Telecom Bretagne/Institut Mines-Telecom/UeB/ Lab-STICC, Technopôle Brest-Iroise, Brest 29238, France (e-mail: samir.saoudi@telecom-bretagne.eu).

Digital Object Identifier 10.1109/TPWRD.2012.2203616
In this paper, we focus on the design of an uplink random-access scheme for an OFDMA-based indoor PLC network. The carrier sense multiple access with collision avoidance (CSMA/CA) technology-based random access is defined as one solution at the PLC medium-access control (MAC) layer in [2] to support best-effort applications and applications that rely on prioritized quality of service (QoS). In OFDMA-based random-access schemes, each station (STA) in an indoor PLC network is allowed to maintain its own backoff counter and contend with other STAs to access the available subchannels. Therefore, the system performance is possibly improved in this manner of simultaneous multiple transmissions. Besides, OFDMA systems are especially robust against narrowband disturbances and impulsive noise encountered in the PLC networks and, thus, OFDMA offers a suitable multiple-access candidate for PLC networks.

However, the PLC line grids are inherently susceptible to attenuation, noise, and interference. They cause PLC channel-state variations in frequency and time domains, and ultimately impact the system performance greatly. In addition, the difficulty with uplink transmissions is that they cannot avoid contentions unless a user specifically has a channel dedicated to it. The contention procedure induces collisions, especially under heavily loaded conditions, as well as idle slots, which degrade the transmission efficiency. Consequently, the design of a channel-aware access scheme that exploits multiuser diversity across subchannels and at time instants is a key problem for PLC systems in order to achieve efficient transmissions. We pay attention to the uplink data traffic over PLC networks in this paper. Accordingly, the objective of our access scheme design is to enhance the system throughput, which is crucial to performance measurement of data traffic.

Motivated by the fact that different channels at different time instants experience different channel conditions, great attention has been devoted to the opportunistic access to enhance the system performance. An opportunistic Aloha for the uplink transmissions in OFDMA wireless networks is presented in [3]. Therein, a set of subchannels is allocated to a contending user under the condition that its obtained subchannel gains derived from the instantaneous channel-state information (CSI) are greater than the defined threshold. If a collision occurs, the collided subchannel set is not utilized by the contending users. This work gives the subchannel assignment solution from the opportunistic viewpoint, but collision resolution is not taken into account. Chang et al. [4] put forward a collision resolution policy for the opportunistic Aloha scheme of [3], where collided good subchannels are set with a random backoff time for future retrial, rather than discarded. Furthermore, a backoff mechanism is shown in [5], where all users estimate their channel power gains and compare them with a predefined 
backoff threshold. The users with greater channel gain experience fewer numbers of backoff slots, which results in higher priority of the channel contention. Therefore, they are more likely to obtain their favorable subchannels. In [6], another MAC protocol is proposed for OFDMA and CSMA/CA PLC networks. Similarly, the backoff time is decreased with the increasing gain of throughput for each user. Theses works provide the subchannel allocation or collision resolution schemes in the frequency domain, while the impact of multiuser diversity in the time domain on system performance is not discussed.

Compared with the aforementioned schemes, some randomaccess schemes are studied to take advantage of multiuser diversity in subchannel allocation and channel contention procedures. In [7] and [8], a backoff mechanism for OFDMA and CSMA/CA systems is presented. In this mechanism, the backoff counter is initialized as a random value between 0 and the minimum contention window size for the first attempt, then decreased by the number of available subchannels per backoff slot in order to minimize the collision probability. Nevertheless, the mechanism of the backoff counter decrement cannot guarantee that subchannels are assigned to the users with the best channel quality. For example, a smaller value of the backoff counter is distributed to a "worse" user (inversely for a "good" user), and their backoff counters are reduced by the same value. Therefore, the subchannel allocation for the different users is not optimized in this case, which leads to the degradation of the overall system throughput.

Our scheme design aims at exploiting multiuser diversity in the frequency and time domains during the channel allocation and contention processes. The basic idea is to reduce the collision probability and idle slots during the contention procedure by opportunistically assigning subchannels to the STAs with better channel conditions. In this paper, we first consider the design of an opportunistic random-access scheme in an OFDMAbased multiuser PLC system. By exploiting multiuser diversity, the proposed scheme adjusts the backoff time for each active user according to its own channel-state variations; moreover, it assigns users their corresponding favorable subchannels for transmissions at the end of contention procedures to further improve the system throughput. Subsequently, we analyze the average system throughput of the proposed scheme. On the basis of analytical results, we find the optimal settings of the proposed scheme that will maximize the system throughput, assuming that the number of users and subchannels are known. In addition, a large set of simulations is performed to compare our proposed scheme with the CSMA/CA in [2] and other opportunistic multichannel schemes in [5] and [8] in terms of average system throughput, collision probability, and fairness among users.

The remainder of this paper is organized as follows. Section II presents the employed system model. The proposed opportunistic random-access scheme for OFDMA-based indoor PLC systems is described in Section III. In Section IV, we give the theoretical analysis about the system throughput as well as the related optimization of parameters. The simulation results are shown and analyzed in Section V. Section VI concludes this paper.

\section{SySTEM MODEL}

An OFDMA-based home PLC network with $D$ subcarriers and $M$ users is taken into account in this paper. These users act

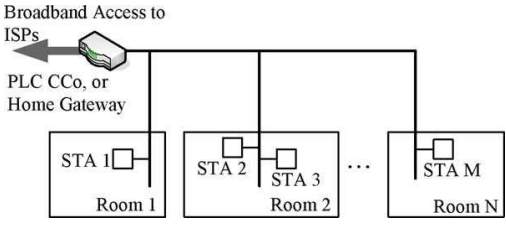

Fig. 1. Topology of a home PLC network.

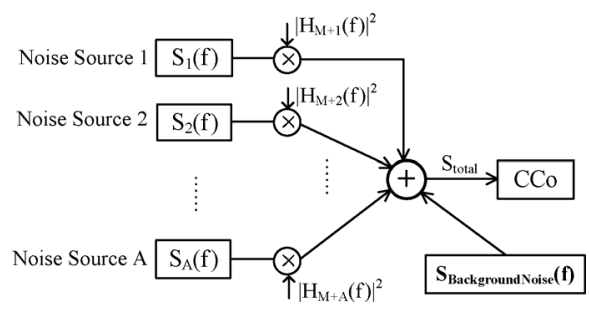

Fig. 2. Illustration of noise modeling.

as base communication devices (i.e., STAs) and connect with the central coordinator (CCo), or Home Gateway (Fig. 1). The CCo executes external broadband communications with an internet service provider (ISP), and coordinates data transmissions within the home. By utilizing the OFDMA technique, multiple STAs can obtain different subchannels and communicate with the CCo simultaneously.

The uplink multipoint-to-point transmissions (i.e., from STAs to the $\mathrm{CCo}$ ) are considered in this paper. The connection between each STA and CCo is defined as a path, and the channel transfer function of path $i$ is denoted by $H_{i}$. We assume that each STA operates independently and has knowledge of only its own instantaneous channel conditions. When the signal is transferred on one path, noise at the CCo can be regarded as a combination of background noise and noises generated by all close-by electrical appliances connected to the power-line network, as shown in Fig. 2. $S_{i}$ is defined as the noise variance generated by the $i$ th path, so the contribution of the $i$ th noise resource to the CCo is $S_{i} \times\left|H_{i}\right|^{2}$. Employing the noise model in [9], it is assumed that the paths from 1 to $M$ correspond to the PLC communication paths, and the remaining paths from $(M+1)$ to $(M+A)$ represent the noise sources. Since STAs operate independently, the aggregated noise signals are assumed to be uncorrelated, and the noise variance at the CCo is expressed as

$$
S_{\mathrm{CCo}}=\sum_{i=1}^{A} S_{i} \times\left|H_{M+i}\right|^{2} .
$$

Here, the noise power spectral density (PSD) varies in different time frames, so the noise power spectrum at the CCo is time varying.

In [10]-[12], the authors have investigated and confirmed the cyclostationary nature of noise in power lines. The noise power spectrum is observed to be periodic with the frequency of the power line cycle $(50$ or $60 \mathrm{~Hz}$ ). Thereby, the channel signal-to-noise ratio (SNR) exhibits periodicity characteristics. Accordingly, the cyclostationary PLC channel noise is modeled in [13] using a time mask multiplied by the noise PSD [i.e., $\left.S_{i}(t)=S_{i} \times \operatorname{Mask}(t)\right]$. The time mask spans in an ac cycle period $(20 \mathrm{~ms}$ for $50 \mathrm{~Hz}$ ), and shows how the noise PSD varies 


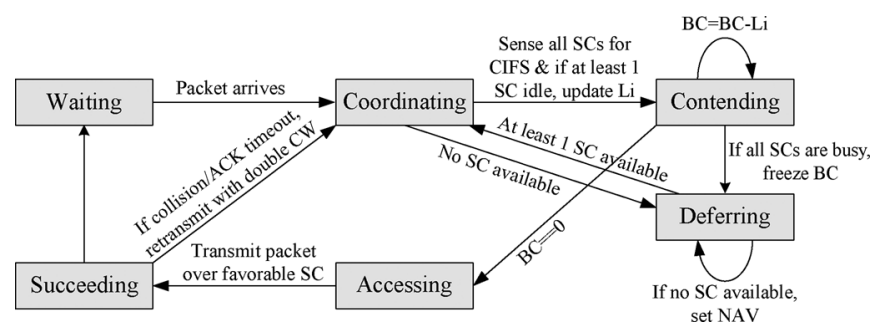

Fig. 3. State transition diagram of the proposed opportunistic scheme, where $\mathrm{SC}, \mathrm{BC}$, and NAV are denoted as the subchannel, backoff counter, and network allocation vector, respectively.

from one time slot to another during the 20-ms cycle period. By repeating the time mask over every ac power cycle, the cyclostationary channel is established. Consequently, the SNR value over path $i$ from $\mathrm{STA}_{i}$ to the CCo is computed as

$$
\mathrm{SNR}_{i}=\frac{\left|H_{i}\right|^{2}}{S_{\mathrm{CCo}}} .
$$

Based on the aforementioned SNR values, the transmission rate at the PHY layer can be obtained by employing the bitloading algorithm for OFDM-based PLC channels [14]. The bit loading is realized over 64 subcarriers selected in $1.8-30-\mathrm{MHz}$ frequency interval, where the number is shown as an example and does not affect the behavior of the proposed scheme. The 64 selected subcarriers are contiguously divided into groups of subchannels due to the fact that subcarriers which fall within the coherence bandwidth have similar responses. Each subchannel contains the same number of subcarriers.

It is assumed that all STAs and CCo are synchronized with the ac cycle in order to exploit the cyclostationary nature of PLC channel SNR.

\section{OpportUNiSTIC SCHEME BASED ON THE DYNAMIC BACKOFF TIME}

In this section, a novel random-access scheme with dynamic backoff mechanism is proposed. Therein, the backoff counter is adaptively decremented for each active STA according to the channel-state variations in the frequency and time domains to reduce collision probability. Moreover, subchannels are assigned to the users that can achieve more benefits of data rate from these subchannels to improve the system throughput.

We assume that $M$ stations in the network want to transmit data frames to the CCo by contending over $K$ subchannels, and each STA knows its own channel conditions. The state transition diagram of the proposed scheme is shown in Fig. 3. When a data frame arrives at $\mathrm{STA}_{i}, \mathrm{STA}_{i}$ will detect the states of all subchannels for a contention interframe spacing (CIFS) period. If there is no idle subchannel, $\mathrm{STA}_{i}$ will persist in monitoring the channel. On the contrary, if at least one subchannel is available, $\mathrm{STA}_{i}$ initializes the contention window $(\mathrm{CW})$ size, the backoff counter, and the backoff decrement step, and then enters into the backoff procedure.

The CW size of $\mathrm{STA}_{i}, W_{i}$ is initialized as $W_{\min }$ at its first transmission attempt. This value is determined by the binary exponential backoff algorithm after each unsuccessful transmission until it reaches $W_{\max }$

$$
W_{i}=2^{N_{\text {attempt }}-1} W_{\min }
$$

where $N_{\text {attempt }}$ is the number of transmission attempts. The initial value of the backoff counter $B C_{i}$ is set as $\left(W_{i}-1\right)$ for all STAs in order to demonstrate the advantage of the proposed opportunistic scheme. Subsequently, the backoff counter is reduced by a backoff decrement step per backoff slot during the contention period if there is at least one idle subchannel per backoff slot. Here, the backoff decrement step of $\mathrm{STA}_{i}$ is denoted as $L_{i}\left(L_{i} \geq 1\right)$, and it is designed to take channel-state variations into account as follows:

$$
L_{i}=\left\lceil\frac{\max _{j \in\{1,2, \cdots, k\}}\left(r_{i}^{j}\right)}{r_{r e f}}\right\rceil
$$

where $\lceil\cdot\rceil$ denotes the ceiling function, $\max \left(r_{i}^{j}\right)$ represents the maximum data rate achieved by $\mathrm{STA}_{i}$ over the idle subchannel $j$ at the beginning of its corresponding backoff process ( $j$ is defined as $\mathrm{STA}_{i}$ 's favorable subchannel in this case), $k$ is the number of idle subchannels at the beginning of the current time slot, and $r_{\text {ref }}$ is introduced as a reference value. $r_{\text {ref }}$ is optimized in Section IV-B.

During $\mathrm{STA}_{i}$ 's backoff process, three cases may be encountered as follows.

1) If $\mathrm{STA}_{i}$ 's favorable subchannel remains idle during the backoff process, its backoff counter is reduced by $L_{i}$, derived from (4) per backoff slot until its value reaches zero.

2) If $\mathrm{STA}_{i}$ 's favorable subchannel is occupied by another STA, and there is still at least one subchannel available, $\mathrm{STA}_{i}$ will update $L_{i}$ based on the conditions of the available subchannels according to (4), and then continue its backoff procedure.

3) If $\mathrm{STA}_{i}$ senses that the subchannels, which are idle within the precedent backoff slot, are all occupied by other STAs in the current backoff slot, it will interrupt the current backoff procedure, and freeze the backoff counter. Subsequently, $\mathrm{STA}_{i}$ will monitor the channel until there is at least one subchannel available for a duration of CIFS, reset $L_{i}$ according to the state of the available subchannels and continue the backoff process in the same way as before.

It should be noted that collisions are resolved in the frequency and time domains by allocating different subchannels to different STAs and adjusting $L_{i}$ according to (4). Meanwhile, the idle slots during the contention period are reduced compared with the conventional protocol. Besides, the backoff policy always entitles the STAs with better instantaneous channel conditions to obtain higher priority in the assignment of their favorable subchannels and, thus, the system throughput is improved.

When the backoff counter of $\mathrm{STA}_{i}$ reaches zero, it launches the transmission to the CCo by utilizing the chosen subchannel during its backoff process. If the CCo receives the data frame from $\mathrm{STA}_{i}$ successfully, it will reply to $\mathrm{STA}_{i}$ with an acknowledgement $(\mathrm{ACK})$ message after a response interframe spacing 


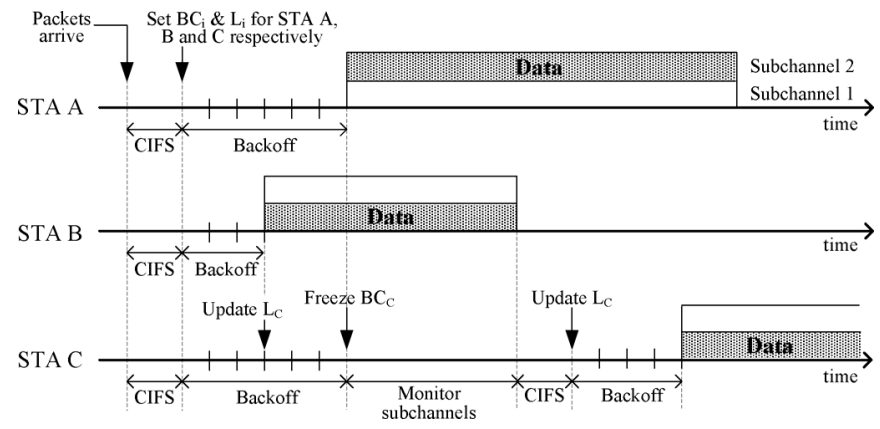

Fig. 4. Illustration of the proposed opportunistic CSMA/CA protocol for OFDMA-based indoor PLC systems.

(RIFS) period. However, if $\mathrm{STA}_{i}$ does not receive the acknowledgement from the CCo within a predefined deferred period, it will regard this transmission as disabled. $\mathrm{STA}_{i}$ will free this subchannel utilized for transmission after receiving its corresponding ACK from the CCo or after this predefined period elapses in the case of unsuccessful transmissions.

The transmission failures originate from channel transmission errors or frame collisions when more than one STA starts to transmit simultaneously over the same subchannel. In this paper, we only consider the failures caused by frame collisions since the system throughput is greatly affected by collisions when the number of contending STAs increases. Upon collisions, the collided STAs will update their corresponding CW sizes according to the binary exponential algorithm and try to retransmit the current frame in the subsequent contention period by repeating the above process. The maximum number of transmission attempts is set as 3 for all STAs. If $\mathrm{STA}_{i}$ cannot receive the ACK from the CCo after three transmission attempts, it will drop this frame.

Fig. 4 illustrates the aformentioned protocol with an example of three STAs and two subchannels. Without loss of generality, data frames are supposed to arrive at these three STAs simultaneously. They will set their own $\mathrm{CW}$ sizes and related backoff decrement steps according to (4) after a CIFS period. STA A and B finish their backoff procedures in six and three backoff slots, respectively, based on their actual channel conditions in frequency and time domains. Subsequently, they select the corresponding favorable subchannels (i.e., subchannel 2 for STA A and 1 for STA B) to transmit data frames.

Here, we assume that STA C's favorable subchannel is 1. It is noted that subchannel 1 is occupied by STA B during STA C's backoff procedure; therefore, STA C has to update its backoff decrement step. When there is no available subchannel, STA C will begin to monitor the channel until sensing at least one idle subchannel, then will restart the backoff procedure.

\section{ThroughPUt ANALYSIS AND OptimizATION}

We consider the saturated throughput scenario in this work (i.e., all STAs have infinite data frames to transmit), which is a fundamental performance measurement for data traffic. In this section, the saturated system throughput of the proposed scheme is analyzed. Based on the analytical results, we optimize the related parameters of the proposed scheme in order to maximize the system throughput.

\section{A. Theoretical Analysis}

In the context of saturated throughput, the average system throughput depends on the number of STAs and the average throughput of each subchannel when the system is in steady state. In the following text, we analyze the average system throughput in two cases: 1) when the number of STAs is greater than that of subchannels (i.e., $M>K$ ) and 2) $M \leq K$, respectively.

1) When $M>K$ : We assume that the data rate over each subchannel is independent and identically distributed (i.i.d.) for all STAs. Hence, the average system throughput is determined by

$$
\bar{\rho}_{\text {sum }}=K \cdot \bar{\rho}_{1}
$$

where $\bar{\rho}_{1}$ is the average throughput of one subchannel. Under the condition that there are $k$ idle subchannels at a certain slot during the transmission period, $\bar{\rho}_{1}$ is calculated by

$$
\bar{\rho}_{1}=\sum_{k=1}^{K} \operatorname{Pr}\left(K_{\text {idle }}=k\right) \cdot \bar{\rho}_{1}(k)
$$

where $\operatorname{Pr}\left(K_{\text {idle }}=k\right)$ is the steady-state probability that there are $k$ idle subchannels at a certain slot, and $\bar{\rho}_{1}(k)$ is the mean throughput of one subchannel among these $k$ idle subchannels. In the following text, we consider the derivation of $\bar{\rho}_{1}(k)$ and $\operatorname{Pr}\left(K_{\text {idle }}=k\right)$, respectively.

The probability density function (PDF) of each STA's data rate over each subchannel is denoted by $f(r)$, where $r$ is the corresponding data rate. Here, $f(r)$ is modeled by using the method of curve fitting based on the channel parameters in Section II. More details about the related curve-fitting process can be found in [15]. The cumulative distribution function (CDF) of $r$ is defined as $F(r)=P(R \leq r)$.

We suppose that $\mathrm{STA}_{i}$ achieves its maximum data rate $(v)$ over one subchannel among $k$ idle subchannels $(1 \leq k \leq K)$ and, thus, the corresponding PDF of $v$ is calculated by

$$
g(k, v)=\left(\begin{array}{c}
k \\
1
\end{array}\right) \cdot f(v) \cdot F^{k-1}(r)
$$

where $F^{k-1}(r)$ denotes the probability that the achievable rates over $(k-1)$ idle subchannels are lower than $v . F^{k-1}(r)$ is given by

$$
F^{k-1}(r)=(P(R \leq r))^{k-1} .
$$

Accordingly, we substitute $\max \left(r_{i}^{j}\right)$ by $v_{i}$ in (4) for the sake of convenience in the following analysis, and (4) is rewritten as

$$
L_{i}=L\left(v_{i}\right)=\left\lceil\frac{v_{i}}{r_{\mathrm{ref}}}\right\rceil
$$

where $L$ is a function of $v$.

In our proposed scheme, one subchannel is assigned to $\mathrm{STA}_{i}$ that obtains its best channel condition over this subchannel. Therefore, the time duration of one successful transmission cycle (without collisions) of $\mathrm{STA}_{i}$ is obtained as follows:

$$
\begin{array}{r}
T_{s}\left(W_{i}, v_{i}\right)=T_{\mathrm{CIFS}}+\left\lceil\frac{W_{i}-1}{L\left(v_{i}\right)}\right\rceil \cdot T_{\mathrm{slot}}+\frac{N b_{\text {frame }}}{v_{i}} \\
+T_{\mathrm{RIFS}}+T_{\mathrm{ACK}}
\end{array}
$$


where $T_{\mathrm{CIFS}}, T_{\mathrm{RIFS}}, T_{\mathrm{ACK}}, T_{\text {slot }}$, and $N b_{\text {frame }}$ represent the duration of CIFS, RIFS, ACK, backoff slot, and number of bits of a data frame, respectively. $T_{\mathrm{CIFS}}, T_{\mathrm{RIFS}}$, and $T_{\text {slot }}$ are set to the same values as [2], described in Section V. It should be noted that the item $\left(\left\lceil\left(W_{i}-1\right) / L\left(v_{i}\right)\right\rceil \cdot T_{\text {slot }}\right)$ is the actual backoff time that $\mathrm{STA}_{i}$ experiences before starting its transmission process. Here, $W_{i}$ is doubled at each unsuccessful transmission, while the retransmission probability is low in the case where the number of STAs does not greatly exceed that of the subchannels. Moreover, this item is very small compared to the frame transmission time $\left(N b_{\text {frame }} / v_{i}\right)$. Thus, we ignore the impact of increasing $W_{i}$ on $T_{s}$ in (10) due to retransmissions for analysis purposes. Consequently, $W_{i}$ is approximated to be uniformly distributed in the range of $\left(1, W_{\min }\right)$ in the steady state. Then, we can derive the throughput during one successful transmission cycle described in (10) as follows:

$$
\gamma_{\mathrm{tx}}\left(W_{i}, v_{i}\right)=\frac{N b_{\text {frame }}}{T_{s}\left(W_{i}, v_{i}\right)} .
$$

Now, let us consider the probability of one successful transmission over one subchannel in case of collisions. If there are $k$ idle subchannels at a certain slot, $(M-K+k)$ STAs will contend with each other during the subsequent backoff period. In the proposed scheme, we can efficiently avoid collisions by optimizing the values of $W$ and $r_{\text {ref }}$ in (9). Hence, the collisions over the busy subchannels are neglected for the purpose of analysis. We suppose that $m$ STAs among these $(M-K+k)$ STAs obtain their corresponding maximum data rates over these $k$ subchannels and start to contend. For one of the $k$ subchannels (e.g., $\beta$ ), a successful transmission over subchannel $\beta$ implies that the achievable rate of one STA among the $m$ STAs over $\beta$ is greater than that of the remaining $(m-1)$ STAs; moreover, no collisions occur between this STA and the $(m-1)$ STAs. We first calculate the probability that this STA does not collide with one STA among these $(m-1)$ STAs.

In the proposed backoff mechanism, the backoff counter of each STA is decremented by $L\left(v_{i}\right)$ according to (9). During the transmission period, if some subchannels are released, the STAs that have data frames to transmit will begin to backoff over the idle subchannels simultaneously. Therefore, if a collision occurs between two STAs that wait to transmit over subchannel $\beta$, for instance, $\mathrm{STA}_{i}$ and $\mathrm{STA}_{j}, \mathrm{STA}_{i}$ 's backoff time during the contention period is equal to $\mathrm{STA}_{j}$ 's, which means that

$$
\left\lceil\frac{W_{i}-1}{L\left(v_{i}\right)}\right\rceil=\left\lceil\frac{W_{j}-1}{L\left(v_{j}\right)}\right\rceil .
$$

In the case where $W_{i}$ is different from $W_{j}$, it is obvious that different values of $L$ may also satisfy (12). We should note that when $W_{i}=W_{j}$, different values of $L$ may also satisfy (12) since the value of the backoff counter decrement step is expressed in a ceiling function in (9). For example, when $W_{i}=$ $W_{j}=32$ and the values of $L$ of three STAs are 8, 9, and 10, respectively, they will experience the same backoff time (i.e., four backoff slots during the contention period). Here, we define the number of backoff slots that $\mathrm{STA}_{i}$ experiences during the backoff period as follows:

$$
N_{i}^{\text {backoff }}\left(W_{i}, v_{i}\right)=\left\lceil\frac{W_{i}-1}{L\left(v_{i}\right)}\right\rceil .
$$

On the contrary, if $\mathrm{STA}_{i}$ does not collide with other contending STAs, its corresponding value of $N_{i}^{\text {backoff }}$ is lower than that of the other contending STAs.

Given that $\mathrm{STA}_{i}$ obtains its maximum data rate over $\beta$, the probability that $\mathrm{STA}_{i}$ can successfully choose subchannel $\beta$ ( $\beta \in\{1,2, \cdots, k\})$ without colliding with one STA among $(m-1)$ is calculated by

$$
\begin{aligned}
\operatorname{Pr}_{\text {no_col_l }}\left(W_{i}, v_{i}\right)= & \operatorname{Pr}\left(N_{i}^{\text {backoff }}<N_{j}^{\text {backoff }}\right), \\
& j \in\{1, \ldots, i-1, i+1, \ldots, m\} .
\end{aligned}
$$

Here, $\operatorname{Pr}_{\text {no_col_1 }}\left(W_{i}, v_{i}\right)$ in (14) is obtained by accumulating all probabilities that any STA collides with $\mathrm{STA}_{i}$. For details, please refer to [15].

Accordingly, the probability that $\mathrm{STA}_{i}$ does not collide with these $(m-1)$ STAs over $\beta$ is

$$
\operatorname{Pr}_{\text {no_col }}\left(W_{i}, v_{i}\right)=\left(\operatorname{Pr}_{\text {no_col_1 } 1}\right)^{m-1} .
$$

Since $r$ is assumed to be i.i.d., the corresponding maximum value $(v)$ over each subchannel is thus i.i.d. Furthermore, the CW size of each STA is approximated as uniform distribution. As a result, (15) is rewritten as (16) to indicate the probability that one STA among $m$ contending STAs does not collide with the remaining $(m-1)$ ones as follows:

$$
\operatorname{Pr}_{\text {no_col }}(W, v)=\left(\operatorname{Pr}_{\text {no_col_1 } 1}\right)^{m-1} .
$$

Similarly, the throughput during one successful transmission cycle in (11) is rewritten as

$$
\gamma_{\mathrm{tx}}(W, v)=\frac{N b_{\text {frame }}}{T_{s}(W, v)} .
$$

Therefore, the probability that one STA among $m$ contending STAs can successfully transmit with the corresponding maximum data rate $(v)$ is expressed as

$$
\operatorname{Pr}_{\text {success }}(k, m, W, v)=\left(\begin{array}{c}
m \\
1
\end{array}\right) \cdot g(k, v) \cdot \operatorname{Pr}_{\text {no_col }}(W, v) \text {. }
$$

Based on (18), on the condition that there are $k$ idle subchannels and $m$ contending STAs, the average throughput of the "successful STA" (with the CW size as $W$ ) over its favorable subchannel is calculated by

$$
\bar{\rho}_{3}(k, m, W)=\int_{0}^{\infty} \gamma_{\mathrm{tx}}(W, v) \cdot \operatorname{Pr}_{\text {success }}(k, m, W, v) d v .
$$

In our proposed scheme, the $\mathrm{CW}$ sizes are initialized as $W_{\min }$ for all STAs, and are approximated to be uniformly distributed within the range of $\left(1, W_{\min }\right)$. Therefore, given that there are $k$ idle subchannels and $m$ contending STAs, the mean throughput of one subchannel is given by

$$
\bar{\rho}_{2}(k, m)=\sum_{W=1}^{W_{\min }} \frac{1}{W_{\min }} \cdot \bar{\rho}_{3}(k, m, W) .
$$

Consequently, the average throughput of one subchannel among $k$ idle subchannels is determined by

$$
\bar{\rho}_{1}(k)=\sum_{m=1}^{M-K+k} \frac{\left(\begin{array}{c}
M-K+k \\
m
\end{array}\right) \cdot(k-1)^{M-K+k-m}}{k^{M-K+k}} \cdot \bar{\rho}_{2}(k, m) .
$$


Now, we consider the probability of $k$ idle subchannels in the steady state (i.e., $\operatorname{Pr}\left(K_{\text {idle }}=k\right)$ ). We define $K_{\text {idle }}(t)$ as the corresponding stochastic process. Motivated by [7], we utilize the Markov chain to model the process $K_{\text {idle }}(t)$. In order to determine the transition probability, we denote $X(k \mid M-K+k)$ as the number of events that $(M-K+k)$ STAs contend over $k$ subchannels $(k \geq 1)$. The value of $X(k \mid M-K+k)$ is calculated by the following iterative expression:

$X(k \mid M-K+k)$

$=\left\{\begin{array}{l}1, \quad \text { if } k=1 \\ k^{M-K+k}-\sum_{j=1}^{k-1}\left(\begin{array}{c}k \\ j\end{array}\right) \cdot X(j \mid M-K+k),\end{array}\right.$

otherwise.

In (22), $k$ is a positive integer. We define that $X(0 \mid M-K)=$ 0 and $X(0 \mid 0)=1$. Here, we assume that the backoff time is much less than the data frame transmission time. Subsequently, under the condition that $k$ subchannels are available, the probability that $k^{\prime}$ subchannels will be selected for the subsequent transmissions is

$$
\begin{aligned}
\operatorname{Pr}\left(K_{\mathrm{slt}}^{\text {new }}\right. & \left.=k^{\prime} \mid K_{\text {idle }}=k\right) \\
& \equiv\left(\begin{array}{c}
k \\
k^{\prime}
\end{array}\right) \cdot \frac{X(k \mid M-K+k)}{k^{M-K+k}}, \quad 0 \leq k^{\prime} \leq k .
\end{aligned}
$$

Let $T_{\mathrm{tx}}(v)$ be the transmission time of a data frame over one subchannel, then $T_{\mathrm{tx}}(v)$ is equal to $N b_{\text {frame }} / v$. Accordingly, the probability that the transmission of one data frame finishes at a certain slot is

$$
\operatorname{Pr}_{\text {tx_slot }}(v)=\frac{1}{\left\lceil\frac{T_{\text {tx }}(v)}{T_{\text {slot }}}\right\rceil} .
$$

Given that $k$ subchannels are idle, the probability that $k^{\prime}$ subchannels among $(M-k)$ busy subchannels will become idle at a certain slot is given by

$$
\begin{gathered}
\operatorname{Pr}\left(K_{\text {idle }}^{\text {new }}=k^{\prime} \mid K_{\text {idle }}=k\right) \equiv\left(\begin{array}{c}
M-k \\
k^{\prime}
\end{array}\right) \cdot\left(\operatorname{Pr}_{\text {tx_slot }}(v)\right)^{k^{\prime}} \\
\cdot\left(1-\operatorname{Pr}_{\text {tx_slot }}(v)\right)^{M-k-k^{\prime}}, \quad 0 \leq k^{\prime} \leq K-k .
\end{gathered}
$$

Consequently, the transition probability between any two states $\left(K_{\text {idle }}=k\right)$ and $\left(K_{\text {idle }}=l\right)(0 \leq k, l \leq K)$ is calculated on the basis of (23) and (25), as shown in (26) at the bottom of the page. According to (26), we can now determine the steady-state probability of $\operatorname{Pr}\left(K_{\text {idle }}=k\right)$.

2) When $M \leq K$ : If one STA finishes its transmission and releases its utilized subchannel at a certain slot, $(K-M+1)$ subchannels will be idle at this slot. That is to say, this STA can select its favorable subchannel from these $(K-M+1)$ subchannels to execute its subsequent backoff process. Based on this point, we assume that there are no collisions among $M$ STAs. Then, the average system throughput is given by

$$
\bar{\rho}_{\text {sum }}=M \cdot \bar{\rho}_{1 S T A}
$$

where $\bar{\rho}_{1 S T A}$ is the average throughput of one STA. It is obtained based on the PDF of the maximum data rate from (7) and the mean throughput during one successful transmission cycle from (11) as follows:

$$
\bar{\rho}_{1 S T A}=\sum_{W=1}^{W_{\min }} \frac{1}{W_{\min }} \cdot \int_{0}^{\infty} \gamma_{\mathrm{tx}}(W, v) \cdot g(K-M+1, v) d v .
$$

\section{B. Parameter Optimization}

In this paper, we are more interested in the average system throughput [i.e., $\bar{\rho}_{\text {sum }}$ in (5) or (27)] for the performance measurement of best-effort traffic. The problem of optimizing $\bar{\rho}_{\text {sum }}$ is thus formulated as follows:

$$
\begin{array}{ll}
\text { Maximize : } & \bar{\rho}_{\text {sum }} \\
\text { Subject to : } & W>1, \quad L \geq 1, \quad M>1, \quad K>1, \\
& W, L, M \text { and } K \text { are integers. }
\end{array}
$$

We assume that the number of STAs $(M)$ and subchannels $(K)$ are known, and $\bar{\rho}_{\text {sum }}$ is essentially a function of $W$ and $r_{\text {ref }}$. According to (12) and (14), we deduce that the impacts of the $\mathrm{CW}$ size $(W)$ and $r_{\text {ref }}$ on the average system throughput are not independent; thus, the optimization is aimed at the combina-

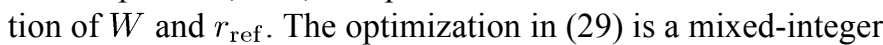
nonlinear programming (MILNP) problem. Here, the branchand-bound algorithm [16] is adopted to solve this optimization problem.

In the case where $M>K$, frame collisions have a great impact on the system throughput. However, thanks to our opportunistic design and the optimization of the $\mathrm{CW}$ size $(W)$ and $r_{\text {ref }}$, the collisions of the proposed scheme can be efficiently avoided. Moreover, increasing the values of $W$ of different STAs, and simultaneously decreasing $r_{\text {ref }}$ contribute to reducing the collision probability and apparently to enhancing the system throughput. For $M>K$, the optimization results of $\left(W, r_{\text {ref }}\right)$ are shown in Table I in the cases of different values of $K$, where $r_{\text {ref }}$ and $\rho_{\mathrm{sum}}^{\mathrm{opt}}$ are in the unit of bits per second and megabits per second, respectively.

On the other hand, when $M \leq K$, the main benefit of decreasing $W$ and increasing $r_{\text {ref }}$ is to diminish the idle slots before transmissions since the collision probability is very small. However, the contribution of reducing the idle slots to

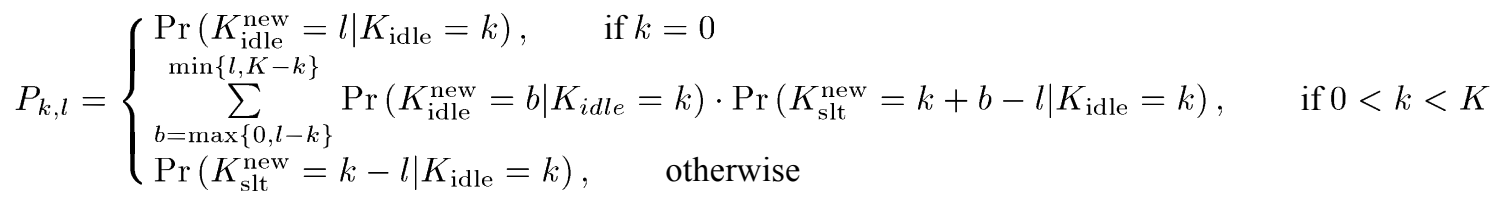


TABLE I

System Throughput Versus Optimal $\left(W, r_{\text {ref }}\right)$ When $M>K$

\begin{tabular}{|c|c|c|c|c|c|c|c|c|}
\hline \multirow{2}{*}{$M$} & \multicolumn{2}{|l|}{$K=2$} & \multirow{2}{*}{$M$} & \multicolumn{2}{|l|}{$K=4$} & \multirow{2}{*}{$M$} & \multicolumn{2}{|l|}{$K=8$} \\
\hline & $W, r_{\text {ref }}$ & $\rho_{\text {sum }}^{\text {opt }}$ & & $W, r_{\text {ref }}$ & $\rho_{\text {sum }}^{\text {opt }}$ & & $W, r_{r e f}$ & $\rho_{\text {sum }}^{o p t}$ \\
\hline 3 & $540,7.2 \times 10^{4}$ & 2.80 & 5 & $540,4.8 \times 10^{4}$ & 3.06 & 9 & $540,2.4 \times 10^{4}$ & 3.36 \\
\hline 5 & & 2.93 & 7 & & 3.24 & 12 & & 3.63 \\
\hline 7 & & 2.87 & 9 & & 3.25 & 14 & & 3.78 \\
\hline
\end{tabular}

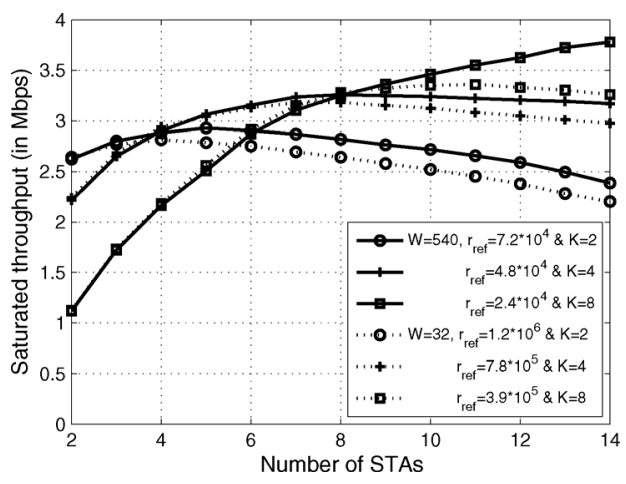

Fig. 5. Optimization of $W$ and $r_{\text {ref }}$ of the proposed scheme with respect to the number of STAs.

the system throughput is very weak since the backoff duration is much less than the transmission time. In order to demonstrate the impact of decreasing the idle slots before transmissions on the system throughput when $M \leq K$, we set $\left(W, r_{\text {ref }}\right)$ as (32, $\left.1.2 \times 10^{6}\right),\left(32,7.8 \times 10^{5}\right)$ and $\left(32,3.9 \times 10^{5}\right)\left(r_{\text {ref }}\right.$ is in the unit of bits per second) for $K=2,4$, and 8 , respectively. The corresponding results of analytical throughput are shown in Fig. 5 and are compared with the results by using the configurations in Table I for $M>K$. For each $K$, we note that the difference of system throughput based on these two configurations is small when $M \leq K$. Furthermore, with the decreasing $W$ and increasing $r_{\text {ref }}$, the obtained gain of throughput when $M \leq K$ is greatly inferior to the loss of throughput in the case where $M>K$. For example, for $K=4$ and $M=3, \bar{\rho}_{\text {sum }}$ is augmented by $1.32 \%$ compared with that of Table I; whereas $\bar{\rho}_{\text {sum }}$ of $M=8$ is decreased by $3.71 \%$. Since we focus more on the throughput when $M>K$, we utilize the optimal $\left(W, r_{\text {ref }}\right)$ in Table $\mathrm{I}$ in simulations in Section V due to their distinct improvement of system throughput when $M>K$.

\section{Simulation Results}

The performance of the proposed opportunistic random-access scheme for OFDMA-based systems is evaluated through computer simulations in this section.

The simulations are executed in 100 ac power cycles $(2 s)$. For the simulation parameters, the duration of CIFS, RIFS, and the backoff slot are set as $35.84,26$, and $35.84 \mu \mathrm{s}$, respectively, according to [2]. Each arrival frame size is set as $1000 \mathrm{~B}$. At the PHY layer, the channel transfer functions are obtained by the measurement described in [17]. The 64 selected subcarriers are contiguously grouped into $K$ subchannels, and each subchannel contains the same number of subcarriers. In addition, we assume that all STAs have frames with the same priority.

In the simulations, we increase the number of STAs from 2 to 14 , and each scenario is conducted for 500 independent

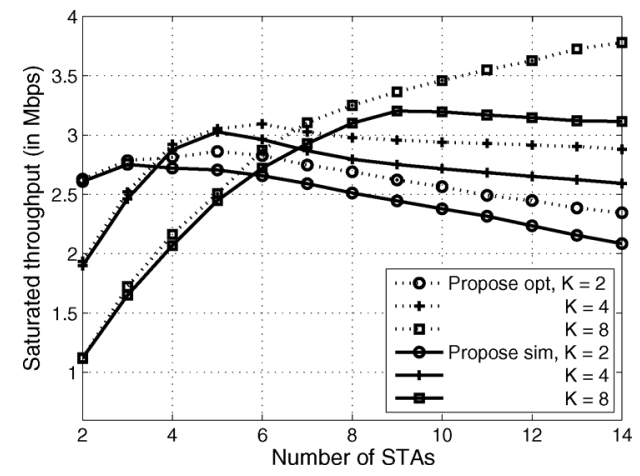

Fig. 6. Comparison of the system throughput of the proposed scheme between analytical and simulation results.

simulation runs in order to obtain an average value. For our proposed scheme, the analytical results in Section IV and the corresponding simulation results are marked as "Propose opt" and "Propose sim", respectively. These results are in terms of average system throughput, collision probability, and fairness among STAs, and they are compared with the related results of other schemes in the literature. The following two schemes are given as benchmarks.

1) HPAV CSMA/CA: the CSMA/CA protocol in [2] based on single-channel access;

2) Kwon Opp: the opportunistic scheme in [8] where the decrement of the backoff counter is determined by the number of available subchannels at the current instant.

\section{A. Average Throughput}

Fig. 6 shows the analytical results of the saturated throughput of the proposed scheme and the corresponding simulation results with respect to the number of STAs $(M)$ when the number of subchannels $(K)$ is equal to 2,4 , and 8 . We note that both types of throughput results do not drop sharply with the increasing $M$ due to multiuser diversity. In addition, the analytical results are slightly superior to the simulation results, and this overestimation is caused by the fact that the data rate over $K$ subchannels is approximated as i.i.d. for all STAs in the analysis of Section IV. This approximation can balance a quantity of "worse" subchannels, which tends to contribute to the overestimated throughput. However, the data rates in reality are not i.i.d.

The average system throughput is compared between the simulation results of the proposed scheme and other schemes in Fig. 7. When the number of STAs is fewer than that of subchannels (i.e., $M<K$ ), the saturated system throughput is augmented with the increasing number of $M$ for each case of $K$ in [8] and the proposed scheme. While for a given $M$, the throughput is decreased with the rising $K$ since the available 


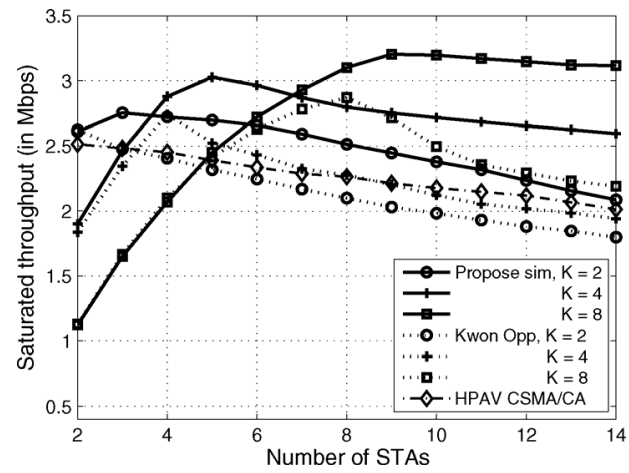

Fig. 7. Comparison of the system throughput with respect to the number of STAs.

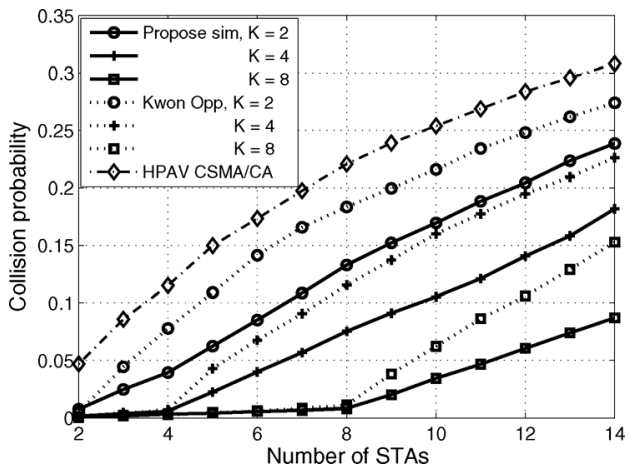

Fig. 8. Comparison of the average collision probability with respect to the number of STAs.

subchannels are not fully employed. For example, in the case of $M=2$, the saturated throughput of $K=4$ is higher than that of $K=8$.

On the other hand, when $M \geq K$, the saturated throughput is dramatically lowered with the rise of $M$ for each $K$ in the scheme of [8] because the random backoff counter value of this scheme cannot guarantee the STAs to obtain their favorable subchannels. In contrast to it, the proposed scheme obtains a minor deterioration in the system throughput when increasing $M$ for each $K$ because the multiuser diversity is exploited in both backoff and subchannel allocation processes. Moreover, for a certain $K$, the greater $M$ is, the more gain the proposed scheme can achieve from the multiuser diversity. Besides, the throughput remains around $2.6 \mathrm{Mb} / \mathrm{s}$ as $K=4$ and $M \geq 10$ because the proposed system could not obtain more gain from the multiuser diversity with considerably greater $M$.

It should be noted that the throughput of HPAV CSMA/CA [2] degrades with increasing $M$ because of collisions.

\section{B. Average Collision Probability}

In Fig. 8, the comparison of the average collision probability is demonstrated when increasing the number of STAs in the case of different $K$. For a given $K$, our proposed scheme outperforms the other two schemes in terms of collision probability. Further, with the increase of $M$, the proposed scheme obtains lower collision probabilities when $K$ is greater. For example, when $K=8$, the maximum collision probability of the proposed scheme is lower than $10 \%$, which results from more achieved gains thanks to multiuser diversity.

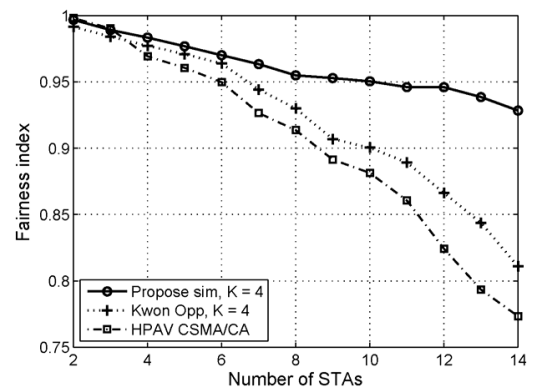

(a)

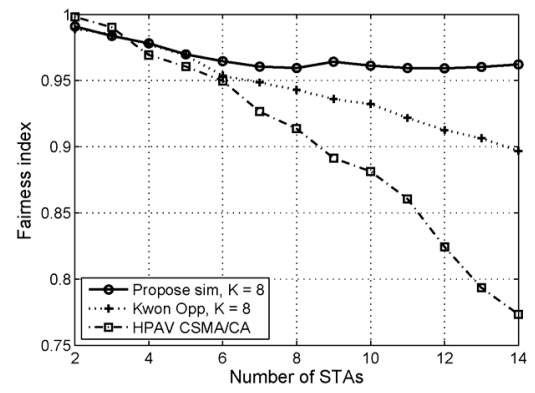

(b)

Fig. 9. Comparison of the average fairness index with respect to the number of STAs.

\section{Fairness Among STAs}

In opportunistic schemes, the users with better channel conditions are always scheduled preferentially in order to achieve high throughput. On the contrary, the users with an unfavorable channel state may be starved in some successive slots. Therefore, the tradeoff between high throughput and fairness is significant to opportunistic systems. Consequently, we analyze the fairness issue of the proposed scheme.

Jain's fairness index [18] is utilized to measure the fairness among STAs

$$
\text { fairness index }=\frac{\left(\sum_{i=1}^{M} \rho_{i}\right)^{2}}{M \cdot \sum_{i=1}^{M} \rho_{i}^{2}}
$$

where $\rho_{i}$ is the sum throughput of $\mathrm{STA}_{i}$ during the simulation round. The more this fairness index approaches the value of 1 , the fairer the scheme is.

In Fig. 9, we note that the multichannel access schemes, that is, [8] and the proposed scheme bring higher fairness compared with the single-channel access scheme (HPAV CSMA/CA [2]). With the increasing number of STAs $(M)$, the average fairness index of the proposed scheme is better than that of [8] due to the designed backoff mechanism. Moreover, for a given $M$, a greater number of subchannels $(K)$ can bring higher fairness since the $M$ STAs have more opportunities to transmit.

\section{CONCLUSION}

This paper aims to design a random-access scheme for OFDMA-based indoor PLC networks in the context of multiuser and multichannel. Motivated by the fact that PLC channels vary in frequency and time domains, we propose an opportunistic scheme based on adaptive backoff time, where the backoff counter is decremented by exploiting the knowledge of channel-state variations for each active STA in order to reduce 
the collision probability and idle slots before transmissions. Furthermore, subchannels are assigned to the STAs with better channel conditions for transmissions at the end of contention procedures to further enhance the system throughput.

For the proposed scheme, we utilize an analytical system throughput model to achieve the optimal parameter settings, assuming that the number of STAs and subchannels are known. Simulation results demonstrate that by using the optimal parameters, the proposed scheme highly reduces the collision probability, and achieves a significant improvement of the global throughput. Meanwhile, the fairness of the proposed scheme remains comparable to other single-channel and multichannel access schemes.

\section{REFERENCES}

[1] A. Majumder and J. Caffery, Jr., "Power line communications," IEEE Potentials, vol. 23, no. 4, pp. 4-8, Oct./Nov. 2004.

[2] HomePlug Powerline Alliance, HomePlug AV Specif., Ver. 1.1 May 2007.

[3] K. Bai and J. Zhang, "Opportunistic multichannel Aloha: Distributed multiaccess control scheme for OFDMA wireless networks," IEEE Trans. Veh. Technol., vol. 55, no. 3, pp. 848-855, May 2006.

[4] Y.-J. Chang, F.-T. Chien, and C. C. J. Kuo, "Opportunistic access with random subchannel backoff (OARSB) for OFDMA uplink," in Proc. IEEE Int. Global Telecommun. Conf., 2007, pp. 3240-3244.

[5] D. Wang, H. Minn, and N. Al-Dhahir, "A distributed opportunistic access scheme and its application to OFDMA systems," IEEE Trans. Commun., vol. 57, no. 3, pp. 738-746, Mar. 2009.

[6] S.-G. Yoon, D. Kang, and S. Bahk, "OFDMA CSMA/CA protocol for power line communication," in Proc. IEEE Int. Symp. Power Line Commun. App., 2010, pp. 297-302.

[7] H. Kwon, H. Seo, S. Kim, and B. G. Lee, "Generalized CSMA/CA for OFDMA systems: Protocol design, throughput analysis, and implementation issues," IEEE Trans. Wireless Commun., vol. 8, no. 8, pp. 4176-4187, Aug. 2009.

[8] H. Kwon, S. Kim, and B. G. Lee, "Opportunistic multi-channel CSMA protocol for OFDMA systems," IEEE Trans. Wireless Commun., vol. 9, no. 5, pp. 1552-1557, May 2010.

[9] H. Meng, Y. L. Guan, and S. Chen, "Modeling and analysis of noise effects on broadband power-line communications," IEEE Trans. Power Del., vol. 20, no. 2, pt. 1, pp. 630-637, Apr. 2005.

[10] M. Katayama, T. Yamazato, and H. Okada, "A mathematical model of noise in narrowband power line communication systems," IEEE J. Sel. Areas Commun., vol. 24, no. 7, pp. 1267-1276, Jul. 2006.

[11] J. A. Cortes, F. J. Cañete, L. Diez, and J. T. Entrambasaguas, "Characterization of the cyclic short-time variation of indoor power-line channels response," in Proc. IEEE Int. Symp. Power Line Commun. App., 2005, pp. 326-330.

[12] F. J. C. Corripio, J. A. C. Arrabal, L. D. del Rio, and J. T. E. Munoz, "Analysis of the cyclic short-term variation of indoor power line channels," IEEE J. Sel. Areas Commun., vol. 24, no. 7, pp. 1327-1338, Jul. 2006.

[13] A. Chowdhery, S. Jagannathan, J. M. Cioffi, and M. Ouzzif, "A polite cross-layer protocol for contention-based home power-line communications," presented at the IEEE Int. Conf. Commun., Dresden, Germany, Jun. 14-18, 2009.

[14] J. M. Cioffi, "EE379C - Advanced Digital Communication," 2007/2008. [Online]. Available: http://www.stanford.edu/group/cioffi/ ee379c/
[15] R. Dong, "Cross-layer optimization for indoor powerline communication networks," Ph.D. dissertation, Dept. Signal Commun., TELECOM Bretagne, Brest, France, 2011.

[16] D. Li and X. L. Sun, Nonlinear Integer Programming. New York: Springer, 2006.

[17] Conexant Systems Inc., Gigle Semiconductor, Intel Corporation, and Intellon Corporation, UIT-T SG15 Contribution-Powerline Channel Data, ANSI Contribution NIPP-NAI-2007-107, Minneapolis, MN, May 2007

[18] R. Jain, D. Chiu, and W. Hawe, "A quantitative measure of fairness and discrimination for resource allocation in shared computer systems," Hudson, CA, Tech. Rep. DEC Res. Rep. TR-301, Sep. 1984.

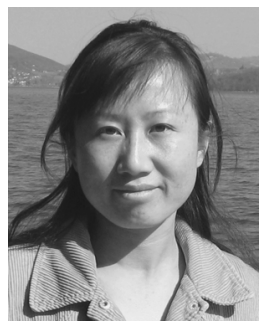

Rongping Dong (S'10) received the M.S. degree in telecommunication and networks from the Institut National des Sciences Appliquées (INSA) de Lyon, Lyon, France, in 2008, and the Ph.D. degree in signal and telecommunication from Telecom Bretagne, Brest, France, in 2011.

Currently, she is a Postdoctoral Research Fellow with the Université de Bretagne-Sud, Lorient, France. Her research interests include crosslayer optimization and design, resource allocation, as well as media-access control and scheduling in multichannel-based networks.

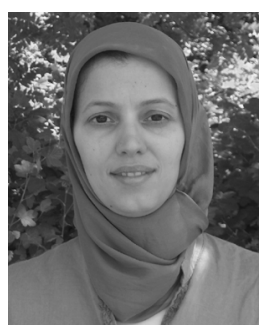

Meryem Ouzzif (M'09) received the M.S. and Ph.D. degrees in electrical engineering from the Institut $\mathrm{Na}$ tional des Sciences Appliquées (INSA) de Rennes, Rennes, France, in 2000 and 2004, respectively.

Currently, she is a Network Architect and Project Manager at Orange Labs, Cesson-Sevigne, France. Her current field of interest is related to interaccess mobility and distributed architectures. Prior to that, she was a Research Engineer in advanced digital communications (multiple-input, multiple-output, crosslayer optimization, orthogonal frequency-division multiplexing) for wireline optical, DSL, and power-line communication transmissions. In 2002, she was a Visiting Researcher at the Information Systems Laboratory, Stanford University, Stanford, CA, with Prof. John Cioffi.

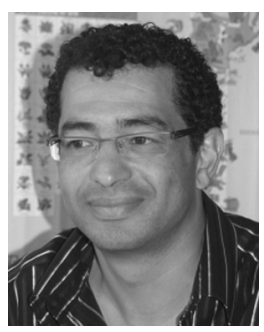

Samir Saoudi (M'01-SM'09) received the Ph.D. degree in telecommunications from Rennes-I University, Rennes, France, in 1990, and the Habilitation à Diriger des Recherches en Sciences at Telecome Bretagne in 1997.

$\mathrm{He}$ is qualified as an Electrical and Electronics Engineer with Telecom Bretagne, Brest, France, in 1987. Since 1991, he has been with the Signal and Communications Department, Telecom Bretagne, where he is currently a Full Professor and Leader of Digital Communications Group, Lab-STICC. In 2009 , he spent three months on sabbatical stay in Orange Labs-Tokyo, as a Visiting Professor. His research interests include speech/audio coding, nonparametric probability density function estimation, code-division multiple-access techniques, multiuser detection, and multiple-input multiple-output techniques. His teaching interests are signal processing, probability, stochastic processes, and speech processing.

Prof. Saoudi has been the General Chairman of the 2nd International Symposium ISIVC 2004, and Technical Co-Chair of the MIMO Systems Symposium at IWCMC 2010. Since 2006, he has been a member of the Board of Directors, French Telecom Institute. 